\title{
Numerical Methods for Chemically Reacting Fluid Flow Computation under Low-Mach Number Approximation
}

\author{
Toshiyuki ARIMA
}

Honda R\&D Co., Ltd./Chuo University

(Communicated by S. Miyoshi)

\begin{abstract}
A mathematical model of environmental fluid is presented to describe fluid flow motions with large density variations. Moreover the associated numerical methods are discussed. The model of environmental fluid is formulated as an unsteady low-Mach number flow based on the compressible Navier-Stokes equations. For low-Mach number flows, the acoustic effects are assumed to be weak relative to the advection effects. Under this assumption, detailed acoustic effects can be removed from governing equations. The low-Mach number formulation thus enables numerical flow analysis with a projection methodology that uses high-order accurate upwind difference of the convection terms with a time step restricted solely by an advection Courant-Friedrichs-Lewy (CFL) condition. The algorithm presented here is based on an iterative implicit time evolution of second order accuracy and a high-accurate spatial discretization with TVD properties for unsteady low-Mach number flows. It is seen from the results on the verification for test cases of flows with a wide range of density variations that our numerical method is validated.
\end{abstract}

\section{Introduction}

In the compressible Navier-Stokes equations, two kinds of propagation speeds are involved, namely, the convective velocities of fluid particles and the sound velocity which is the propagation speed of sound waves. In fact, it is known that the application of numerical methods for the compressible Navier-Stokes system to low-speed flows does not necessarily provide us with satisfactory information regarding the convergence. This fact suggests that numerical simulations are insufficient and the associated computational results turn out to be inaccurate. These numerical difficulties are caused by the circumstances that the characteristic velocities in the compressible Navier-Stokes systems are the convective speed and sound speed, and so that their ratios become large and the so-called stiffness of the system may occur due to the disparity of eigenvalues of the system.

One of the efficient counter measures for those numerical difficulties is to induce a system of equations for incompressible flows from the compressible Navier-Stokes equations and make analysis under the assumption that the density does not change in both space and time. However, in actual applications, even if the convective speed of fluid is a low speed, it is necessary to take the change of density due to the change of temperature into consideration.

Received November 5, 2004 
For instance, mixing processes of air and fuel in engines, water currents in the ocean or air flows in the atmosphere are caused by the nonuniform distribution of temperature as well as thermal stratification, and the air flow field in a neighborhood of a fire spot as well as diffusion of smoke are typical phenomena which take place in environmental fluids and are understood to be compressible Navier-Stokes flows.

One of the efficient counter measures for treating the change of density due to that of temperature is to employ the so-called Boussinesq approximation which asserts that the ratio of the change in density to that of temperature, $\partial \rho / \partial T$, is sufficiently small. Under Boussinesq approximation it is possible to think of buoyancy terms depending upon change in temperature. However, the incompressibility condition is assumed as the equation of continuity. This is inconsistent with the conservation law of mass, and so it is necessary to examine the limitation of such approximations in their applications.

On the other hand, A. Majda proposed a method for deriving the governing equations for the case of low-Mach numbers from the compressible Navier-Stokes equations by using an asymptotic expansion with respect to Mach number which is supposed to be a sufficiently small quantity. Although this method can not be applied to the flows with shocks that are directly related with the propagation of sound waves, the method itself is effective for the analysis of density driven flows associated with a large scale change of temperature and is applicable to a wider variety of fluid phenomena than those under Boussinesq approximations.

The objective of this paper is threefold: First we introduce low-Mach number approximations in accordance with Majda's idea. We next review the formulation of the Navier-Stokes equations with low-Mach number approximations. Thirdly, we extend Najm's numerical method [9] for computation of low-Mach number flows to make the numerical computation stable for higher Reynolds number flows. Furthermore, our new method is verified through the results of numerical simulations for the following three test cases:

(1) Lid-driven cavity flows in incompressible fluid,

(2) Natural convection in a square cavity in case of flows of small density variation,

(3) Premixed combustion of hydrogen and bromine mixture in case of flows of large density variation.

In Section 2 a complete system of the governing equations for fluid flows involving chemical reactions is presented. In Section 3 a low-Mach number model is introduced for isentropic gases as a special case of the Euler system. In Section 4 our model is applied to the Navier-Stokes equations for reacting flows. In section 5 our numerical methods are discussed. Najm's numerical method proposed in [9] is reviewed and then an iterative implicit method with a TVD properties is proposed. In Section 6, computations for the three test cases mentioned above are performed in order to validate our numerical methods. Finally, some conclusions are given. 


\section{Governing equations}

The starting point of our argument is to formulate the governing equations for the fluid phenomena under consideration. In this section, a complete system of governing equations for fluid flows involving chemical reactions is presented. We derived those equations by applying low-Mach number approximations.

2.1. Conservative form of equations for reacting flows. Equations describing chemically reactive flows with $\mathrm{N}$ participating species in conservative formulation are stated as follows:

- Mass Conservation for Chemical Species:

$$
\frac{\partial \rho Y_{i}}{\partial t}+\nabla \cdot\left(\rho Y_{i} \mathbf{v}\right)=-\nabla \cdot \mathbf{j}_{i}+w_{i} \quad(i=1,2, \cdots N)
$$

- Mass Conservation for Mixture Gases:

$$
\frac{\partial \rho}{\partial t}+\nabla \cdot(\rho \mathbf{v})=0
$$

- Conservation of Momentum:

$$
\frac{\partial \rho \mathbf{v}}{\partial t}+\nabla \cdot(\rho \mathbf{v} \otimes \mathbf{v})=-\nabla p+\nabla \cdot \boldsymbol{\tau}+\rho \sum_{i}^{N} Y_{i} \mathbf{f}_{i}
$$

- Conservation of Energy

$$
\begin{gathered}
\rho \frac{\partial \rho e_{t}}{\partial t}+\nabla \cdot\left\{\left(\rho e_{t}+p\right) \mathbf{v}\right\}=-\nabla \cdot \mathbf{q}+\nabla \cdot(\boldsymbol{\tau} \cdot \mathbf{v})+\rho \sum_{i}^{N} Y_{i} \mathbf{f}_{i} \cdot \mathbf{v}+\sum_{i}^{N} \mathbf{f}_{i} \cdot \mathbf{j}_{i} \\
e_{t}=h-\frac{p}{\rho}+\frac{1}{2} \mathbf{v} \cdot \mathbf{v}
\end{gathered}
$$

- Thermodynamic Equation of State:

$$
p=\rho R T M^{-1}, \quad M=\left(\sum_{i=1}^{N} Y_{i} / M_{i}\right)^{-1},
$$

where $\rho$ means the density, $\mathbf{v}$ denotes the velocity vector, $p$ stands for the pressure, $\boldsymbol{\tau}$ represents the viscous tensor, $\mathbf{f}_{i}$ means the body force per unit mass of species $i, Y_{i}$ represents the mass fraction of chemical species $i, \mathbf{j}_{i}$ denotes the diffusive flux vector of species $i, w_{i}$ stands for the mass production rate of species $i, e_{t}$ is the total energy, $\mathbf{q}$ denotes the heat flux vector, $h$ represents the enthalpy, $R$ is the universal gas constant, $T$ denotes the temperature, $M$ means the mean molecular mass, and $M_{i}$ stands for the molecular mass of species $i$. The viscous stress tensor $\boldsymbol{\tau}$, the diffusive flux vector of species $\mathbf{j}_{i}$, and the heat flux vector $\mathbf{q}$ will be given in the section of "Constitutive equation". 
To be consistent with mass conservation, the species mass fractions, the diffusion velocities and chemical sources must satisfy

$$
\begin{aligned}
& \sum_{i}^{N} Y_{i}=1, \quad 0 \leq Y_{i} \leq 1 \\
& \sum_{i}^{N} \mathbf{j}_{i}=0 \\
& \sum_{i}^{N} w_{i}=0 .
\end{aligned}
$$

Note that summation of conservations equations for all species in (1) implies total mass conservation, (2), so that one of those $N+1$ equations is redundant.

2.2. Constitutive equations. The viscous stress tensor, diffusive flux vector, and heat flux vector are modeled by means of the following constitution equations:

- Viscous stress tensor

$$
\boldsymbol{\tau}=\mu\left\{\left(\nabla \mathbf{v}+(\nabla \mathbf{v})^{T}\right)-\frac{2}{3}(\nabla \cdot \mathbf{v}) \mathbf{I}\right\}
$$

The viscous coefficient $\mu$ is obtained by semi-empirical formulae due to Wilke [13] and modified by Bird, et al. [1].

$$
\mu=\sum_{i=1}^{N} X_{i} \mu_{i}\left(\sum_{j=i}^{N} X_{j} \Phi_{i, j}\right)^{-1}
$$

where

$$
\Phi_{i j}=1 \quad(i=j), \quad \Phi_{i j}=\frac{1}{\sqrt{8}}\left(1+\frac{M_{i}}{M_{j}}\right)^{-\frac{1}{2}}\left[1+\left(\frac{\mu_{i}}{\mu_{j}}\right)^{\frac{1}{2}}\left(\frac{M_{j}}{M_{i}}\right)^{\frac{1}{4}}\right]^{2}, \quad i \neq j
$$

and $X_{i}$ represents mole fraction of species $i$ in the mixture:

$$
X_{i}=M M_{i}^{-1} Y_{i}
$$

\section{- Diffusive flux vector of species}

$$
\mathbf{j}_{i}=\rho \mathbf{V}_{i} Y_{i}
$$

where $\mathbf{V}_{i}$ denotes the diffusion velocity of species $i$ and is determined by

$$
\nabla X_{i}=\underbrace{\sum_{j} \frac{X_{i} X_{j}}{D_{i j}}\left(\mathbf{V}_{j}-\mathbf{V}_{j}\right)}_{\text {Stefan-Maxwall }}+\underbrace{\left(Y_{i}-X_{i}\right) \frac{\nabla p}{p}}_{\text {pressure gradient }}+\underbrace{\frac{\rho}{p} \sum_{j} Y_{i} Y_{j}\left(\mathbf{f}_{i}-\mathbf{f}_{j}\right)}_{\text {body force }}
$$




$$
+\underbrace{\sum \frac{X_{i} X_{j}}{\rho D_{i j}}\left(\frac{D_{T, j}}{Y_{j}}-\frac{D_{T, i}}{Y_{i}}\right) \frac{\nabla T}{T}}_{\text {Soret effect }}
$$

where $D_{i j}$ means the binary mass diffusion coefficient matrix and $D_{T, i}$ represents the thermal mass diffusion coefficient for species $i$.

In this paper a form of Fick's law form is employed to evaluate the diffusion velocities of the species in the associated mass-diffusion processes by introducing a diffusion coefficient $D_{i}$.

$$
\mathbf{V}_{i}=-D_{i} Y_{i}^{-1} \nabla Y_{i}
$$

The diffusion coefficients $D_{i}$ are modeled in terms of the binary diffusion coefficient matrix $D_{i j}$ as follows [1].

$$
D_{i}=\left(1-Y_{i}\right)\left(\sum_{i \neq j} D_{i j}^{-1} X_{i}\right)^{-1} .
$$

It turns out that the diffusive flux vector may be modeled as

$$
\mathbf{j}_{i}=-\rho D_{i} \nabla Y_{i},
$$

and the diffusion coefficients $D_{i}$ are modeled in terms of the binary diffusion coefficients $D_{i j}$ as in [1]. Conservation of mass requires that the sum of all $N$ fluxes $\mathbf{j}_{i}$ vanishes (see Eq. (8)) This requirement is not necessarily fulfilled for this diffusion model. A simple remedy is then to subtract any residual from the bulk flow velocity in the species transport equations.

$$
\nabla \cdot\left(\rho Y_{i} \mathbf{v}\right) \rightarrow \nabla \cdot\left\{\rho Y_{i} \mathbf{v}-Y_{i} \sum_{j} \mathbf{j}_{j}\right\}
$$

\section{- Thermal flux vector}

$$
\mathbf{q}=\underbrace{-\lambda \nabla T}_{\text {conduction }}+\underbrace{\sum_{i}^{N} h_{i} \mathbf{j}_{i}}_{\text {mass duffusion }}+\underbrace{R T \sum_{i} \sum_{j} \frac{X_{j} D_{T, i}}{M_{i} D_{i j}}\left(\mathbf{V}_{i}-\mathbf{V}_{j}\right)}_{\text {Dufour effect }}+\underbrace{\mathbf{q}_{R}}_{\text {radiation }}
$$

where $\lambda$ denotes heat conductivity. Dufour effect and thermal radiation are neglected in the present discussions. Hence we have

$$
\mathbf{q}=-\lambda \nabla T+\sum_{i}^{N} h_{i} \mathbf{j}_{i}
$$

The coefficient of heat conductivity of the mixture is obtained through a combination averaging formula [1].

$$
\lambda=\frac{1}{2}\left(\sum_{i=1}^{N} X_{i} \lambda_{i}+\frac{1}{\sum_{i=1}^{N} X_{i} / \lambda_{i}}\right) .
$$


2.3. Enthalpy equation and temperature equation. The equation of energy conservation is usually formulated in term of specific enthalpy through the relation of $e_{t}=$ $h-\frac{p}{\rho}+\frac{1}{2}|\mathbf{v}|^{2}:$

$$
\frac{\partial \rho h}{\partial t}+\nabla \cdot(\rho h \mathbf{v})=\left(\frac{\partial p}{\partial t}+\mathbf{v} \cdot \nabla p\right)-\nabla \cdot \mathbf{q}-\boldsymbol{\tau}: \nabla \mathbf{v}
$$

where the enthalpy is given by means of formation enthalpies $h_{i}$ for each species by

$$
h=\sum_{i=1}^{N} Y_{i} h_{i}
$$

For ideal gases, the enthalpies $h_{i}$ are independent of the pressure, so that the total differential of $h_{i}$ is given in terms of partial heat capacities $C_{p_{i}}$,

$$
d h_{i}=\left(\frac{\partial h_{i}}{\partial T}\right)_{p} d T=C_{p_{i}} d T
$$

The enthalpy $h_{i}$ of a species is given by

$$
h_{i}=h_{0 i}+\int_{T_{0}}^{T} C_{p_{i}} d T,
$$

with an enthalpy $h_{0 i}$ at a reference temperature $T_{0}$. The heat capacity $C_{p}$ of mixture is obtained by summing up all the $C_{p_{i}}$, namely,

$$
C_{p}=\sum_{i=1}^{N} Y_{i} C_{p_{i}}
$$

Combining Equations (23), (24), (25) and (27) together gives

$$
\rho C p\left(\frac{\partial T}{\partial t}+\mathbf{v} \cdot \nabla T\right)=\left(\frac{\partial p}{\partial t}+\mathbf{v} \cdot \nabla p\right)-\nabla \cdot \mathbf{q}-\boldsymbol{\tau}: \nabla \mathbf{v}-\sum_{i}^{N} h_{i} \frac{d\left(\rho Y_{i}\right)}{d t} .
$$

Substituting Eq. (1) into the last term on the right-hand side of Eq. (28), we get

$$
\rho C p\left(\frac{\partial T}{\partial t}+\mathbf{v} \cdot \nabla T\right)=\left(\frac{\partial p}{\partial t}+\mathbf{v} \cdot \nabla p\right)-\nabla \cdot \mathbf{q}-\boldsymbol{\tau}: \nabla \mathbf{v}+\sum_{i}^{N} h_{i}\left(\nabla \cdot \mathbf{j}_{i}-w_{i}\right) .
$$

Substituting of Eq. (21) into Eq. (29) and use of multiplication rule

$$
\nabla \cdot h_{i} \mathbf{j}_{i}-h_{i} \nabla \cdot \mathbf{j}_{i}=\mathbf{j}_{i} \cdot \nabla h_{i}
$$

and

$$
\nabla h_{i}=C p_{i} \nabla T
$$


imply the relation

$$
\begin{aligned}
\rho C p\left(\frac{\partial T}{\partial t}+\mathbf{v} \cdot \nabla T\right)= & \left(\frac{\partial p}{\partial t}+\mathbf{v} \cdot \nabla p\right)+\nabla \cdot(\lambda \nabla T) \\
& +\rho\left(\sum_{i}^{N} C p_{i} \mathbf{j}_{i}\right) \cdot \nabla T-\boldsymbol{\tau}: \nabla \mathbf{v}-\sum_{i}^{N} h_{i} w_{i}
\end{aligned}
$$

2.4. A model of chemical reactions. The parameter $w_{i}$ in the governing equations represents the rate of mass production of species $i$. In order to evaluate this, we need an appropriate model of chemical reactions. Elementary chemical reactions are described as

$$
\sum_{i=1}^{N} v_{i}^{\prime} m_{i} \underset{k_{b}}{\stackrel{k_{f}}{\rightleftarrows}} \sum_{i}^{N} v_{i}^{\prime \prime} m_{i},
$$

where $v_{i}^{\prime} \mathrm{s}$ are stoichiometric coefficients of reactions for educts, $v_{i}^{\prime \prime}$ s are stoichiometric coefficients of reactions for products, $m_{i} \mathrm{~s}$ are the names of the species $i, k_{f}$ stands for reaction rate of forward reaction, and $k_{b}$ means a reaction rate for the backward reaction. The mass production rate $w_{i}$ for species $i$ is computed through the following equation:

$$
w_{i}=M_{i} \sum_{k=1}^{N r}\left(v_{i, k}^{\prime \prime}-v_{i, k}^{\prime}\right) \dot{w}_{k},
$$

where $M_{i}$ denotes the molecular mass of species $i, N_{r}$ means the total number of elementary chemical reaction stages. The symbol $\dot{w}_{k}$ denotes the progress rate of the $k$-th stage of the elementary chemical reaction, and it is calculated as follows:

$$
\dot{w}_{k}=k_{f, k} \prod_{i}^{N} C_{i}^{v_{i, k}^{\prime}}-k_{b, k} \prod_{i}^{N} C_{i}^{v_{i, k}^{\prime \prime}}
$$

where $k_{f, k}$ represents the reaction rate coefficient for forward reaction of the $k$-th stage of the elementary reaction, $k_{b, k}$ is the coefficient of reaction rate for backward reaction of the $k$-th stage of the elementary reaction, and $C_{i}$ is the concentration of species $i$, that is defined as $C_{i}=Y_{i} \rho / M_{i}$. The reaction rate coefficients $k_{f, k}$ and $k_{b, k}$ for the $k$-th elementary reaction are given, respectively, by the Arrhenius-law.

$$
\begin{aligned}
& k_{f, k}=B_{f, k} T^{a_{f}, k} \exp \left(-\frac{E_{f, k}}{R T}\right), \\
& k_{b, k}=B_{b, k} T^{a_{b}, k} \exp \left(-\frac{E_{b, k}}{R T}\right),
\end{aligned}
$$

where the parameters $B_{f, k}$ and $B_{b, k}$ are frequency factors, $\alpha_{f, k}$ and $\alpha_{b, k}$ are temperature indices, and $E_{f, k}$ and $E_{b, k}$ are activation energies of the forward and backward reactions, 
respectively. This law is empirically validated, while the constants are usually determined by experiments.

\section{Low-Mach number approximations for isentropic flows}

For simplicity, the derivation of a low-Mach number model is discussed for the equations for an isentropic gas which is a special case of the Euler system. This reduced model involves the same difficulty as the Navier-Stokes system with low-Mach numbers. It becomes increasingly ill-conditioned as $M_{a} \rightarrow 0$. The simplified system of governing equations for one-dimensional isentropic flows consists of partial difference equations for the density $\rho$, the velocity $v$, and an algebraic equation for the pressure $p$ :

$$
\begin{gathered}
\frac{\partial \rho}{\partial t}+\nabla \cdot(\rho v)=0, \\
\rho \frac{\partial v}{\partial t}+\rho v \nabla \cdot v+\nabla p=0, \\
p=C \rho^{\gamma},
\end{gathered}
$$

together with given the initial conditions

$$
\left.\rho\right|_{t=0}=\rho^{0},\left.\quad v\right|_{t=0}=v^{0} .
$$

Here, the constant $C$ is determined by means of the constant entropy of the system and $\gamma>1$ denotes the ratio of specific heat capacities. The total derivative of the pressure with respect to density is the square of the speed of sound:

$$
c^{2}=\frac{d p}{d \rho}=\gamma \frac{p}{\rho} .
$$

In what follows, it is assumed that the Mach number $M_{a}$, defined by

$$
M_{a}=\frac{v}{c},
$$

be sufficiently small. Since the total derivative of the pressure in terms of the derivative of density is written as

$$
\frac{d p}{d t}=\frac{d p}{d \rho} \cdot \frac{d \rho}{d t}=c^{2} \frac{d \rho}{d t}=c^{2}\left(\frac{\partial \rho}{\partial t}+v \nabla \rho\right),
$$

with the aid of the continuity equation (38). Hence we have

$$
\frac{\partial p}{\partial t}+\rho c^{2} \nabla \cdot v+v \cdot \nabla p=0 .
$$

Therefore, the linearization of the system (38)-(40) takes the form

$$
\frac{\partial u}{\partial t}+A(u) \frac{\partial u}{\partial x}=0,
$$


where $u=(\rho, v, p)^{T}$ and the matrix $A(u)$ is given by

$$
A(v)=\left(\begin{array}{ccc}
v & \rho & 0 \\
0 & v & \rho^{-1} \\
0 & \rho c^{2} & v
\end{array}\right) .
$$

The eigenvalues of $A(u)$ are $\lambda_{1}=v, \lambda_{2,3}=v \pm c$ and correspond to the convective and sound waves, respectively. For low-Mach numbers, the discrepancy between the speed of sound and the convective velocity becomes large, and so $A(u)$ becomes ill-conditioned in the following sense: Greatly varying signal speeds appear in the equations. A time step for the time integration of the equations is adapted to the fastest speed in them. This in turn implies a "stiff" system. In the limit as $c \rightarrow \infty$, the sound waves will propagate infinitely fast, and so the fluid becomes hydrodynamically incompressible in the sense that the fluid compressibility is expressed by $d \rho=(\partial \rho / \partial p) d p+(\partial \rho / \partial T) d T=\left(1 / c^{2}\right) d p+(\partial \rho / \partial T) d T$. In this case, the compressibility of fluid is led only by a thermodynamic effect. First, we introduce nondimensional variables depending on the initial data $v^{0}$ for velocity and $\rho^{0}$ for density:

$$
\tilde{v}=\frac{v}{\max _{\Omega} v^{0}}, \quad \tilde{\rho}=\frac{\rho}{\max _{\Omega} \rho^{0}} .
$$

This lead us to the following non-dimensional form of the Euler equations:

$$
\begin{gathered}
\frac{\partial \tilde{\rho}}{\partial \tilde{t}}+\nabla \cdot(\tilde{\rho} \tilde{v})=0, \\
\tilde{\rho} \frac{\partial \tilde{v}}{\partial \tilde{t}}+\tilde{\rho} \tilde{v} \nabla \cdot(\tilde{v})+\lambda^{2} \nabla p(\tilde{\rho})=0, \\
p(\tilde{\rho})=C \tilde{\rho}^{\gamma},
\end{gathered}
$$

where $\lambda$ means the constant

$$
\lambda=\frac{1}{M_{a} \sqrt{\gamma C}},
$$

and $p(\tilde{\rho})$ is given by Eq. (40):

$$
p(\tilde{\rho})=\frac{p}{\left(\max _{\Omega} \rho^{0}\right)^{\gamma}}=C \tilde{\rho}^{\gamma} .
$$

In the low-Mach number limit, $\lambda$ tends to infinity. Therefore, the pressure gradient in Eq. (49) is multiplied by a large factor. We suppose that asymptotic expansions in term of powers of $\lambda^{-1}$ of the form

$$
\begin{aligned}
& p=p_{0}+\lambda^{-1} p_{1}+\lambda^{-2} p_{2}+O\left(\lambda^{-3}\right) \\
& \tilde{v}=v_{0}+\lambda^{-1} v_{1}+\lambda^{-2} v_{2}+O\left(\lambda^{-3}\right)
\end{aligned}
$$


with certain coefficient functions $p_{j}$ and $v_{i}$. Substituting these expansions into the momentum equation (39) and rearranging the resultant equation with respect to powers of $\lambda$, we obtain

$$
\lambda^{2} \nabla p_{0}+\lambda \nabla p_{1}+\rho \frac{d v_{0}}{d t}+\nabla p_{2}+O\left(\lambda^{-1}\right)=0 .
$$

Comparing the power of $\lambda$, we let $M_{a} \rightarrow 0$ and see that $\nabla p_{0}=\nabla p_{1}=0$. We then call the sum of these pressures, which are constant in space, "thermodynamic pressure",

$$
P_{t h}:=p_{0}(t)+\lambda^{-1} p_{1}(t) .
$$

Therefore, in the low-Mach number approximation, the lower order terms $p_{2}, p_{3}, \cdots$ are neglected in the ideal gas law. The gas law then becomes an equation for the density,

$$
\rho=\left(C^{-1} P_{t h}\right)^{1 / \gamma} .
$$

Majda [8] has shown that under certain smallness condition for the initial data, as $M_{a} \rightarrow 0$, the solution $(p, v)$ of the compressible Euler equation converges to the solution $\left(p_{2}, v_{0}\right)$ of the incompressible Euler equations:

$$
\begin{gathered}
\nabla \cdot v_{0}=0, \\
\rho_{0} \frac{\partial v_{0}}{\partial t}+\rho_{0} v_{0} \cdot \nabla v+\lambda^{-2} \nabla p_{2}=0, \\
\rho_{p}=\left(C^{-1} P_{t h}\right)^{1 / \gamma} .
\end{gathered}
$$

In the incompressible formulation, acoustic wave are suppressed. This is due to the fact that the higher order terms in asymptotic expansions are not taken into account. In the lowMach number approximation, pressure $p$ is decomposed into the thermodynamic pressure $P_{t h}$, which is constant in space, and the "hydrodynamic pressure" $p_{h y d}$, which is caused by fluid motion and neglected in the gas law.

$$
P(x, t)=P_{t h}(t)+p_{h y d}(x, t) .
$$

\section{Navier-Stokes equations for low-Mach numbers}

For the Navier-Stokes equations for reactive flows such that density varies in space due to spatial gradients of temperature and mean molecular mass, a similar low-Mach number approximation can be employed in order to obtain a well-conditioned system. Similarly to the previous case of the Euler equations, the pressure $p$ is split into two parts,

$$
p(x, t)=P_{t h}(t)+p_{\text {hyd }}(x, t),
$$

where the thermodynamic part $P_{t h}$ is constant in space and does not appear in the momentum equation, and the hydrodynamic part $p_{\text {hyd }}$ is neglected in the gas law. In the low-Mach number approximation, the terms describing work due to viscous stress, $\boldsymbol{\tau}: \nabla \mathbf{v}$, and hydrodynamic pressure in the equation for temperature can be neglected. In this study, only gravitation is 
considered as the external force $\mathbf{f}$. Since hydrodynamic pressure $p_{\text {hyd }}$ take several magnitudes smaller than $P_{t h}$, the assumption that the hydrodynamic pressure can be neglected in both equation for temperature and the gas law is in fact appropriate. As a result, the NavierStokes equations for reacting flow are formulated for low-Mach-number approximation in the following way:

- Mass conservation of species

$$
\frac{\partial \rho Y_{i}}{\partial t}+\nabla \cdot\left(\rho Y_{i} \mathbf{v}\right)=\nabla \cdot\left(\rho D_{i} \nabla Y_{i}\right)+w_{i} \quad(i=1,2, \cdots, N)
$$

- Mass conservation

$$
\frac{\partial \rho}{\partial t}+\nabla \cdot(\rho \mathbf{v})=0
$$

- Momentum conservation

$$
\frac{\partial \rho \mathbf{v}}{\partial t}+\nabla \cdot(\rho \mathbf{v} \otimes \mathbf{v})=-\nabla p_{h y d}+\nabla \cdot \boldsymbol{\tau}+\rho \mathbf{g}
$$

- Energy equation

$$
\rho C p\left(\frac{\partial T}{\partial t}+\mathbf{v} \cdot \nabla T\right)=\frac{\partial P_{t h}}{\partial t}+\nabla \cdot(\lambda \nabla T)+\rho\left(\sum_{i}^{N} C p_{i} D_{i} \nabla Y_{i}\right) \cdot \nabla T-\sum_{i}^{N} h_{i} w_{i}
$$

- Thermal state of equation

$$
P_{t h}=\rho \frac{R T}{M}=\rho R T \sum_{i=1}^{N} \frac{Y_{i}}{M_{i}}
$$

Since the material derivative of the thermal state of equation,

$$
\frac{1}{\rho}\left(\frac{\partial \rho}{\partial t}+(\mathbf{v} \cdot \nabla) \rho\right)=\frac{1}{P_{t h}} \frac{\partial P_{t h}}{\partial t} \frac{1}{M}\left(\frac{\partial M}{\partial t}+(\mathbf{v} \cdot \nabla) M\right)-\frac{1}{T}\left(\frac{\partial T}{\partial t}+(\mathbf{v} \cdot \nabla) T\right),
$$

equation (64) is rewritten as

$$
\frac{1}{P_{t h}} \frac{\partial P_{t h}}{\partial t}=-\nabla \cdot \mathbf{v}-\frac{1}{M}\left(\frac{\partial M}{\partial t}+(\mathbf{v} \cdot \nabla) M\right)+\frac{1}{T}\left(\frac{\partial T}{\partial t}+(\mathbf{v} \cdot \nabla) T\right) .
$$

Since $P_{t h}$ is constant in space, the time derivative of $P_{t h}$ is obtained by averaging Eq. (69) over the whole domain $\Omega$.

$$
\begin{aligned}
\frac{\partial P_{t h}}{\partial t} & =-\frac{P_{t h}}{|\Omega|} \int_{\Omega}\left\{\nabla \cdot \mathbf{v}-\frac{1}{T} \frac{d T}{d t}+\frac{1}{M} \frac{d M}{d t}\right\} d x \\
& =-\frac{P_{t h}}{|\Omega|}\left[\int_{\partial \Omega} \mathbf{v} \cdot \mathbf{n} d S+\int_{\Omega}\left\{-\frac{1}{T} \frac{d T}{d t}+\frac{1}{M} \frac{d M}{d t}\right\} d x\right] .
\end{aligned}
$$

Here we consider the case where $\Omega$ is an open domain. The right-hand side of Eq. (70) vanishes and the thermodynamic pressure remains constant in both time and space, independently 
of the assumptions imposed,

$$
P_{t h} \equiv \text { const }
$$

This is our situation that we have to take into account in our application below.

In order to investigate the effect of heat release in chemical reactions in a closed vessel, we suppose that non-slip boundary condition for the velocities $(\mathbf{v}=0$ on $\partial \Omega)$, constant $\Omega$ in time, constant mean molecular mass $M$, and constant specific heat capacity $C_{p}$. In this case the boundary integral in Eq. (70) and time derivative of $M$ vanish. Substituting Eq. (66) into the material derivative of the temperature of Eq. (70) and using the relation Eq. (8), that is, $\sum_{i} \mathbf{j}_{i}=\sum_{i} D_{i} \nabla Y_{i}=0$, and a thermodynamic equation of state in the form $P_{t h} / T=\rho R / M$, we get

$$
\frac{\partial P_{t h}}{\partial t}=\frac{R}{M C_{p}}\left(\frac{\partial P_{t h}}{\partial t}+\frac{1}{|\Omega|} \int_{\Omega} \nabla \cdot(\lambda \nabla T) d x\right) .
$$

For homogeneous Neumann boundary conditions for the temperature on $\partial \Omega$,

$$
\frac{\partial P_{t h}}{\partial t}=\frac{R}{M C_{p}-R} \cdot \frac{1}{|\Omega|} \int_{\Omega}\left(-\sum_{i}^{N} h_{i} w_{i}\right) d x .
$$

For exothermic reaction $\left(w_{i}<0\right)$, the above equation means that thermodynamic pressure increases.

\section{Numerical methods}

We begin by reviewing the low-Mach number pressure correction method proposed by Najm, el al. [9], propose a method for improving the computational efficiency of time integration by introducing implicit iteration method. In the method proposed by Najm el al., so-called Predictor-Corrector method is employed for numerical integration. For both stages of the predictor and corrector, the fractional method is incorporated in accordance with the pressure-split momentum equations. The predictor uses a second order Adams-Bashforth time integration scheme to update the velocity as well as scalar field. A pressure correction step is incorporated in order that the continuity equation holds. The corrector is based on a second order quasi Crank-Nicolson integration and also incorporates a pressure correction step. In their scheme, the rate of change of density in time is derived by differentiating the equation of state Eq. (67) so that the energy equation and the mass conservation of species are combined through the equation of state,

$$
\frac{\partial \rho}{\partial t}=\rho\left[-\frac{1}{T} \frac{\partial T}{\partial t}-M \sum_{i=1}^{N} \frac{1}{M_{i}} \frac{\partial Y_{i}}{\partial t}\right] .
$$

For open domain systems considered in this study, the thermodynamic pressure $P_{t h}$ is uniform and constant so that the material derivative of pressure is equal to zero. 
5.1. Numerical scheme of Najm et al. [9]. The Najm's algorithm is summarized as follows:

\section{Predictor stage:}

1. The time derivative $\partial T /\left.\partial t\right|^{n}, \partial\left(\rho Y_{i}\right) /\left.\partial t\right|^{n},(i=1, \cdots, N-1)$, and $\partial \rho /\left.\partial t\right|^{n}$ are explicitly evaluated through Eq. (66), Eq. (63), and Eq. (74), respectively.

$$
\begin{aligned}
\left.\frac{\partial T}{\partial t}\right|^{n}= & -\left(\mathbf{v}^{n} \cdot \nabla^{n}\right) T^{n}+\frac{1}{\rho^{n} C_{p}^{n}}\left[\nabla \cdot\left(\lambda^{n} \nabla T^{n}\right)\right. \\
& \left.+\rho^{n}\left(\sum_{i}^{N} C p_{i}^{n} D_{i}^{n} \nabla Y_{i}^{n}\right) \cdot \nabla T^{n}-\sum_{i}^{N} h_{i}^{n} w_{i}^{n}\right] \\
\left.\frac{\partial\left(\rho Y_{i}\right)}{\partial t}\right|^{n}= & -\nabla \cdot\left(\rho^{n} Y_{i}^{n} \mathbf{v}^{n}\right)+\nabla \cdot\left(\rho^{n} D_{i}^{n} \nabla Y_{i}^{n}\right)+w_{i}^{n}, \quad i=1,2, \cdots, N-1 \\
\left.\frac{\partial \rho}{\partial t}\right|^{n}= & \rho^{n}\left[-\frac{1}{T^{n}} \frac{\partial T^{n}}{\partial t}-M^{n} \sum_{i=1}^{N} \frac{1}{M_{i}^{n}} \frac{\partial Y_{i}^{n}}{\partial t}\right] .
\end{aligned}
$$

The concentration $\rho Y_{N}$ of the last species is determined from $\sum_{i=1}^{N} \rho Y_{i}=\rho$.

2. Predicted values for the density and scalar concentration field are determined as

$$
\begin{gathered}
\frac{\rho^{P}-\rho^{n}}{\Delta t}=\left.\frac{3}{2} \frac{\partial \rho}{\partial t}\right|^{n}-\left.\frac{1}{2} \frac{\partial \rho}{\partial t}\right|^{n-1} \\
\frac{\rho^{P} Y^{P}-\rho^{n} Y^{n}}{\Delta t}=\left.\frac{3}{2} \frac{\partial\left(\rho Y_{i}\right)}{\partial t}\right|^{n}-\left.\frac{1}{2} \frac{\partial\left(\rho Y_{i}\right)}{\partial t}\right|^{n-1}
\end{gathered}
$$

and the predicted temperature is obtained from the equation of state,

$$
T^{P}=P_{t h} /\left(R \rho^{P}\right) .
$$

3. The intermediate velocity $\hat{\mathbf{v}}$ is determined by integrating the pressure-split momentum equations,

$$
\frac{\rho^{P} \hat{\mathbf{v}}^{P}-\rho^{n} \mathbf{v}^{n}}{\Delta t}=\frac{3}{2} \mathbf{R}^{n}-\frac{1}{2} \mathbf{R}^{n-1}
$$

where

$$
\mathbf{R}=-\nabla \cdot(\rho \mathbf{v} \otimes \mathbf{v})-\nabla \cdot \boldsymbol{\tau}+\rho \mathbf{g} .
$$

4. The intermediate hydrodynamic pressure is determined by solving the pressure Poisson equation. The derivation of the Poisson equation is discussed later.

$$
\nabla^{2} p_{\text {hyd }}^{P}=\frac{1}{\Delta t}\left[\nabla \cdot\left(\rho^{P} \hat{\mathbf{v}}^{P}\right)+\left.\frac{\partial \rho}{\partial t}\right|^{P}\right]
$$


where $\partial \rho /\left.\partial t\right|^{P}$ is given by the second-order discretization, since $\mathbf{v}^{P}$ is not known at this stage, and $\partial \rho /\left.\partial t\right|^{P}$ cannot be directly evaluated from Eq. (64).

$$
\left.\frac{\partial \rho}{\partial t}\right|^{P}=\frac{1}{2 \Delta t}\left(3 \rho^{P}-4 \rho^{n}+\rho^{n-1}\right) .
$$

5. Finally, the predicted velocity $\mathbf{v}^{P}$ is obtained through the projection step:

$$
\frac{\rho^{P} \mathbf{v}^{P}-\rho^{P} \hat{\mathbf{v}}^{P}}{\Delta t}=-\nabla p_{h y d}^{P} .
$$

The pressure Poisson equation (83) is obtained by taking the divergence of Eq. (85) under the restriction of the continuity condition, $\partial \rho^{P} / \partial t+\nabla \cdot\left(\rho^{P} \mathbf{v}^{P}\right)=0$. Adding Eq. (81) to Eq. (85), we get

$$
\begin{aligned}
\frac{\rho^{P} \mathbf{v}^{P}-\rho^{n} \mathbf{v}^{n}}{\Delta t} & =\left.\frac{3}{2}(-\nabla \cdot(\rho \mathbf{v} \otimes \mathbf{v})-\nabla \cdot \boldsymbol{\tau}+\rho \mathbf{g})\right|^{n} \\
& -\left.\frac{1}{2}(-\nabla \cdot(\rho \mathbf{v} \otimes \mathbf{v})-\nabla \cdot \boldsymbol{\tau}+\rho \mathbf{g})\right|^{n-1} \\
& -\nabla p_{\text {hyd }}^{P} .
\end{aligned}
$$

We find that in the predictor stage of the Najm's scheme pressure term is implicitly treated by means of a backward Euler scheme and other terms are explicitly treated using a second order Adams-Bashforth scheme.

\section{Corrector stage:}

1. The time derivative of the density and species concentrations at the $(n+1)$ th time step are calculated using the predicted values. The corrected values are calculated by applying Crank-Nicolson time integration of the second order accuracy.

$$
\begin{gathered}
\frac{\rho^{n+1}-\rho^{n}}{\Delta t}=\frac{1}{2}\left(\left.\frac{\partial \rho}{\partial t}\right|^{n}+\left.\frac{\partial \rho}{\partial t}\right|^{P}\right) \\
\frac{\rho^{n+1} Y_{i}^{n+1}-\rho^{n} Y_{i}^{n}}{\Delta t}=\frac{1}{2}\left(\left.\frac{\partial\left(\rho Y_{i}\right)}{\partial t}\right|^{n}+\left.\frac{\partial\left(\rho Y_{i}\right)}{\partial t}\right|^{P}\right) .
\end{gathered}
$$

The temperature at the $(n+1)$ th time step is obtained from the equation of state (67).

$$
T^{n+1}=P_{t h} /\left(R \rho^{n+1}\right) .
$$

2. The momentum equations are decomposed into a part excluding the pressure gradient (Eq. (90)) and the pressure gradient part (Eq. (94)) in accordance with a fractional step method. The intermediate velocities $\hat{\mathbf{v}}^{n+1}$ are calculated by applying the 
Adams-Bashforth time integration to the former equation (the pressure-split momentum equations).

$$
\frac{\rho^{n+1} \hat{\mathbf{v}}^{n+1}-\rho^{n} \mathbf{v}^{n}}{\Delta t}=\frac{3}{2} \mathbf{R}^{n}-\frac{1}{2} \mathbf{R}^{n-1},
$$

where

$$
\mathbf{R}=-\nabla \cdot(\rho \mathbf{v} \otimes \mathbf{v})-\nabla \cdot \boldsymbol{\tau}+\rho \mathbf{g} .
$$

3. The hydrodynamic pressure at the $(n+1)$ th time step is determined by solving the pressure Poisson equation that is obtained by taking the divergence of Eq. (94) under the restriction of the continuity condition, $\partial \rho^{n+1} / \partial t+\nabla\left(\rho^{n+1} \mathbf{v}^{n+1}\right)=0$.

$$
\nabla^{2} p_{\text {hyd }}^{n+1}=\frac{1}{\Delta t}\left[\nabla \cdot\left(\rho^{n+1} \hat{\mathbf{v}}^{n+1}\right)+\left.\frac{\partial \rho}{\partial t}\right|^{n+1}\right] .
$$

Since $\mathbf{v}^{n+1}$ is not determined in this stage, $\left.(\partial \rho / \partial t)\right|^{n+1}$ can not be directly calculated using Eq. (64). Thus, $\left.(\partial \rho / \partial t)\right|^{n+1}$ is determined as

$$
\left.\frac{\partial \rho}{\partial t}\right|^{n+1}=\frac{1}{2 \Delta t}\left(3 \rho^{n+1}-4 \rho^{n}+\rho^{n-1}\right) .
$$

4. Finally, the velocity $\mathbf{v}^{n+1}$ at the $(n+1)$ th time step is determined by

$$
\frac{\rho^{n+1} \mathbf{v}^{n+1}-\rho^{n+1} \hat{\mathbf{v}}^{n+1}}{\Delta t}=-\nabla p_{\text {hyd }}^{n+1} .
$$

As shown in [9], the predictor itself is conditionally stable for relatively low temperature variation (ratio of the highest temperature to the lowest is in the range of $\sim 2$ ). For higher temperature ratios, incorporation of the corrector step is necessary for the stability.

As we have mentioned in the predictor stage, adding of Eq. (90) to Eq. (94) implies

$$
\begin{aligned}
\frac{\rho^{n+1} \mathbf{v}^{n+1}-\rho^{n} \mathbf{v}^{n}}{\Delta t}= & \left.\frac{3}{2}(-\nabla \cdot(\rho \mathbf{v} \otimes \mathbf{v})-\nabla \cdot \boldsymbol{\tau}+\rho \mathbf{g})\right|^{n} \\
& -\left.\frac{1}{2}(-\nabla \cdot(\rho \mathbf{v} \otimes \mathbf{v})-\nabla \cdot \boldsymbol{\tau}+\rho \mathbf{g})\right|^{n-1} \\
& -\nabla p_{\text {hyd }}^{n+1} .
\end{aligned}
$$

We find that in a way similar to the predictor stage, in the corrector stage as well, the pressure term is implicitly treated by means of a backward Euler scheme and the other terms are explicitly treated using a second order Adams-Bashforth scheme.

5.2. Implicit schemes. As an extension of the original scheme, we incorporate an implicit type treatment. Therefore grid refinements, for instance in the direction normal to the wall, do not limit the time step. We need to use fine grid points near the wall to resolve the 
boundary layer, especially, in case of flow calculations with high Reynolds number. Firstly, we consider an implicit scheme for the following scalar transport equation:

$$
u_{t}+\nabla \cdot \mathbf{f}+d=0 .
$$

Using a trapezoidal time-advanced scheme to discretize the time derivative, we get

$$
\frac{u^{n+1}-u^{n}}{\Delta t}+(1-\theta) \nabla \cdot \mathbf{f}^{n}+\theta \nabla \cdot \mathbf{f}^{n+1}+d=0
$$

This scheme is is an explicit scheme for $\theta=0$, a Crank-Nicolson scheme for $\theta=1 / 2$, and a fully implicit scheme for $\theta=1$. Linearizing Eq. (97) with $\mathbf{f}=\mathbf{a} u$, we get

$$
(1+\Delta t \theta \boldsymbol{\nabla} \cdot \mathbf{a}) u^{n+1}=u^{n}-\Delta t[(1-\theta) \nabla \cdot \mathbf{f}+d]^{n} .
$$

Furthermore, we get the following implicit scheme in the delta form with $u^{n+1}=u^{n}+\delta u^{n}$ in Eq. (98).

$$
(1+\Delta t \theta \boldsymbol{\nabla} \cdot \mathbf{a}) \delta u^{n}=-\Delta t(\boldsymbol{\nabla} \cdot \mathbf{f}+d)^{n} .
$$

In case of steady state calculation, the right-hand side of Eq. (99) corresponds to the residual of Eq. (96), which we want to solve. If the residual approaches zero during the iteration process, the additive $\delta u^{n}$ also goes to zero. Therefore, since the accuracy of numerical solution depends upon the accuracy of of the right-hand side of Eq. (99), we can have the room of the selection of the operator on the left-hand side from the point of view of the numerical stability and the computational cost.

For the unsteady calculation, it is necessary to increase temporal accuracy. Although Eq. (99) is discretized using a second-order accurate Clank-Nicolson scheme, the overall accuracy depends on not only the accuracy of spatial discretization of the right-hand side but also that of the left-hand side. Thus, in order to get high accuracy in time, we use CrankNicolson scheme and incorporate the Newton-Raphson iteration procedure. Replacing $u^{n+1}$ with $u^{k}+\delta u^{k}$, and $\nabla \cdot \mathbf{f}^{n+1}$ with $\nabla \cdot \mathbf{f}^{k}+\nabla \cdot\left(\mathbf{a} \delta u^{k}\right)$, we get the following implicit scheme of delta form for unsteady calculation:

$$
\left(1+\frac{1}{2} \Delta t \nabla \cdot \mathbf{a}\right) \delta u^{k}=-\left(u^{k}-u^{n}\right)-\frac{1}{2} \Delta t\left[\nabla \cdot \mathbf{f}^{n}+\nabla \cdot \mathbf{f}^{k}\right]+d,
$$

where

$$
u^{k+1}=u^{k}+\delta u^{k}
$$

The superscript $k$ refers to the iteration cycle between solution at time steps $n$ and $n+1$, the superscript 0 indicates the initial guess for the first iteration. If the iteration converges in the sense of that the condition $\left|\delta u^{k}\right| \leq \varepsilon$ is satisfied for a certain $k$ and a certain admissible error bound $\varepsilon$, we put $u^{n+1}=u^{k}$. The accuracy of solution is obtained from Eq. (100) and depends on that of the right-hand side. It dose not depend on that of the left-hand side, because the right-hand side of Eq. (100) corresponds to Eq. (97) with $\theta=1 / 2$ provided that $\left|\delta u^{k}\right| \rightarrow 0$. 
The stability condition for this scheme is given by

$$
\frac{1}{2} C F L \leq 1, \quad C F L=|\mathbf{a}| / \min \left(\Delta x_{1}, \Delta x_{2}, \Delta x_{3}\right)
$$

which is understood to be a TVD stability condition.

5.3. Iterative implicit schemes. As seen in Eqs. (86) and (95) in the previous section, the discretization in time of momentum equations is similar to two stages (predictor stage and corrector stage) in Najm's scheme. Furthermore, it seems to be no particular reason that the "only two" specific stages (prediction stage and correction stage) should be introduced in the scheme. Here we propose a implicit iteration procedure that is more robust and stable for flow calculations with high Reynolds numbers.

In our scheme the following temporal discretization is employed. For the momentum equation, the pressure term is discretized through a backward Euler method of the first order accuracy. The other terms are discretized by a Crank-Nicolson method of the second order accuracy.

$$
\frac{\rho^{n+1} \mathbf{v}^{n+1}-\rho^{n} \mathbf{v}^{n}}{\Delta t}=-\nabla \cdot(\rho \mathbf{v} \otimes \mathbf{v})-\nabla \cdot \boldsymbol{\tau}+\rho \mathbf{g}-\nabla p_{h y d}^{n+1} .
$$

This equation is split into predictor and corrector parts using $p_{d y n}^{n+1}=p_{d y n}^{n}+\delta p_{d y n}$.

$$
\begin{gathered}
\frac{\rho^{n+1} \hat{\mathbf{v}}-\rho^{n} \mathbf{v}^{n}}{\Delta t}=-\nabla \cdot(\rho \mathbf{v} \otimes \mathbf{v})-\nabla \cdot \boldsymbol{\tau}+\rho \mathbf{g}-\nabla p_{h y d}^{n} \\
\frac{\rho^{n+1} \mathbf{v}^{n+1}-\rho^{n+1} \hat{\mathbf{v}}}{\Delta t}=-\nabla \delta p_{h y d}^{n} .
\end{gathered}
$$

Here $\hat{\mathbf{v}}$ means a provisional value of the velocity vector, which will be corrected later to account using Eq. (105). Following Eq. (100), the implicit scheme of delta form for Eq. (104) can be written as

$$
\begin{aligned}
& {\left[1+\frac{1}{2} \Delta t\left\{\mathbf{v} \cdot \nabla-\frac{\mu}{\rho} \nabla^{2}\right\}\right] \delta \hat{\mathbf{v}}^{k}=-\left(\mathbf{v}^{k}-\mathbf{v}^{n}\right)+\frac{1}{2}\left(r h s_{m}^{k}+r h s_{m}^{n}\right),} \\
& r h s_{m}=-\Delta t\left[(\mathbf{v} \cdot \nabla) \mathbf{v}+\frac{1}{\rho} \nabla p_{d y n}-\frac{1}{\rho} \nabla \boldsymbol{\tau}-\mathbf{g}\right]
\end{aligned}
$$

where

$$
\delta \hat{\mathbf{v}}^{k}=\hat{\mathbf{v}}^{k+1}-\hat{\mathbf{v}}^{k} .
$$

The linearization of fluxes on the left hand side of Eq. (106) is simplified, because the exactitude of this is not essential for the last solution as described in the previous section. Similar implicit schemes of delta form can be derived for the temperature equation and equations of species concentrations. For the temperature equation, we get

$$
\left[1+\frac{1}{2} \Delta t\left\{\mathbf{v} \cdot \nabla-\frac{\lambda}{\rho C_{p}} \nabla^{2}\right\}\right] \delta T^{k}=-\left(T^{k}-T^{n}\right)+\frac{1}{2}\left(r h s_{T}^{k}+r h s_{T}^{n}\right),
$$




$$
r h s_{T}=-\Delta t\left[\mathbf{v} \cdot \nabla T-\frac{1}{\rho C_{p}}\left\{\nabla \cdot(\lambda \nabla T)+\rho\left(\sum_{i}^{N} C p_{i} D_{i} \nabla Y_{i}\right) \cdot \nabla T-\sum_{i}^{N} h_{i} w_{i}\right\}\right] .
$$

Also, for the equations of species concentrations, we get

$$
\begin{aligned}
& {\left[1+\frac{1}{2} \Delta t\left\{\mathbf{v} \cdot \nabla-D_{i} \nabla^{2}\right\}\right] \delta Y_{i}^{k}=-\left(Y_{i}^{k}-Y_{i}^{n}\right)+\frac{1}{2}\left(r h s_{Y}^{k}+r h s_{Y}^{n}\right),} \\
& r h s_{Y}=-\Delta t\left[\mathbf{v} \cdot \nabla Y_{i}-\frac{1}{\rho} \nabla\left(\rho D_{i} \nabla Y_{i}\right)-\frac{w_{i}}{\rho}\right] .
\end{aligned}
$$

We then make an attempt to describe our procedure of computing a numerical solution at the $(n+1)$ th time step from the solution at $n$th step. In the following, the superscript $n$ refers to the values of solutions that are known from the previous time level, the superscript $k$ stand for the iteration cycle between the solution at time levels $n$ and $n+1$, and the superscript 0 indicates the initial guess for the first iteration $k=0$.

1. As values for the initial guess for the values at the next time level, we set

$$
\rho^{0}=\rho^{n}, \quad \mathbf{v}^{0}=\mathbf{v}^{n}, \quad T^{0}=T^{n}, \quad Y_{i}^{0}=Y_{i}^{n}(i=1, \cdots, N) .
$$

We find from Eq. (103) that this choice of values for the initial guess corresponds to the use of an explicit Euler scheme for the first iteration.

2. Time evolution governed by the species concentration equations is treated with the aid of Eqs. (111) and (112).

3. Time evolution governed by the temperature equation is specified through Eqs. (109) and (110).

4. The density at iteration cycle $k+1$ is obtained using the equation of state.

$$
\rho^{k+1}=P_{t h} /\left(R T^{k+1}\right) \text {. }
$$

5. Provisional values of velocity vectors are computed in accordance with the time evolution of the momentum equations by means of Eqs. (106) and (107).

6. Pressure correction. The hydrodynamic pressure at the iteration level $k+1$ is determined by solving the pressure Poisson equation that is obtained by taking the divergence of Eq. (105) under the restriction of the continuity condition, $\partial \rho^{k+1} / \partial t+\nabla\left(\rho^{k+1} \mathbf{v}^{k+1}\right)=0$ :

$$
\nabla^{2} \delta p_{\text {hyd }}^{k+1}=\frac{1}{\Delta t}\left[\nabla \cdot\left(\rho^{k+1} \hat{\mathbf{v}}^{k+1}\right)+\left.\frac{\partial \rho}{\partial t}\right|^{k+1}\right] \text {. }
$$

Since $\mathbf{v}^{k+1}$ is not determined at this stage, $\left.(\partial \rho / \partial t)\right|^{k+1}$ can not be directly obtained from Eq. (64). The value $\left.(\partial \rho / \partial t)\right|^{k+1}$ is determined by

$$
\left.\frac{\partial \rho}{\partial t}\right|^{k+1}=\frac{1}{2 \Delta t}\left(3 \rho^{k+1}-4 \rho^{n}+\rho^{n-1}\right) .
$$


7. Finally, the velocity $\mathbf{v}^{k+1}$ and pressure $p_{h y d}^{k+1}$ are determined by

$$
\begin{aligned}
& \mathbf{v}^{k+1}=\hat{\mathbf{v}}^{k+1}-\frac{\Delta t}{\rho^{k+1}} \nabla \delta p_{\text {hyd }}^{k+1}, \\
& p_{\text {hyd }}^{k+1}=p_{\text {hyd }}^{k}+\delta p_{\text {hyd }}^{k} .
\end{aligned}
$$

This completes one cycle of the iteration process. If more iterations are required the process should continue from step 2 .

5.3.1. Spatial discretization. As to the discretization of the physical space $\Omega$, we employ a collocated variable arrangement such that the scalar and vector quantities are stored on the same grid points [10]. It is well known that a regular variable arrangement causes the so-called checkerboard oscillation due to the decoupling of the velocity and pressure fields. A staggered variable arrangement has been used to overcome this problem. However, staggered arrangement is not necessarily an appropriate choice for calculation with curvilinear coordinate system. Importance of avoiding the checkerboard oscillation is to couple the velocity and pressure fields through the continuity equation. One possibility of avoiding checkerboard oscillations is to interpolate the velocity field in computing the term $\nabla \cdot(\rho \mathbf{v})$ on the right-hand side of Eq. (115) so that variable arrangement can be staggered arrangement.

The discretization of the non-linear convective term is important from the point of view of numerical stability. When dealing with especially flame propagation problem, it is necessary to treat nonlinear convection terms appropriately in order to avoid the numerical instability arising from the numerical oscillations due to rapid changes of physical quantities, such as temperature, density, and species concentration near the flame surface. For this purpose we introduce a total variation diminishing (TVD) scheme.

For simplicity, we consider the time-dependent convection problem for conservative variable in one space dimension. Conservation equations for the mass, momentum, energy, and species concentrations can be written in equation of the following forms for unknown independent variable $q$ that is a physical quantity per unit mass $\phi$ multiplied by the density $\rho$.

$$
\begin{aligned}
& \frac{\partial q}{\partial t}+\frac{\partial f}{\partial x}=0, \\
& f=v \cdot q .
\end{aligned}
$$

Here $q$ denotes a conserved variable and $q$ is written by $q=\rho \phi, f$ stand for the convective flux, and $v$ represents velocity. In general, it is not possible to make discretization with TVD property for such scalar conservation laws, because the flux $f$ is not a function of only $q$ and depends on $u$ as well. However, if we rewrite Eq. (119) in terms of continuity equation $\partial \rho / \partial t=-\partial(\rho v) / \partial x$ as

$$
\frac{\partial \phi}{\partial t}+v \frac{\partial \phi}{\partial x}=0,
$$

we find that the solution of this equation has TVD property since the solution of $\phi$ of Eq. (121) is constant along curve $d x / d t=u$, which is known as the characteristics equation. This can 
be verified by differentiating $\phi(x, t)$ along the curve to find the rate of change of $\phi$ along the characteristics:

$$
\begin{aligned}
\frac{d}{d t} \phi(x(t), t) & =\frac{\partial}{\partial t} \phi(x(t), t)+\frac{\partial}{\partial x} \phi(x(t), t) x^{\prime}(t) \\
& =\phi_{t}+v \phi_{x}=0 .
\end{aligned}
$$

Therefore, it is possible to construct a TVD scheme starting with Eq. (121). In fact, we consider the following equation that is equivalent to Eq. (121).

$$
\frac{\partial \phi}{\partial t}+\frac{\partial(v \phi)}{\partial x}-\phi \frac{\partial v}{\partial x}=0 .
$$

Since the second term is of the derivative of flux, we can incorporate the discretization with TVD property. This will be introduced next.

We discretize the $x-t$ plane by choosing a mesh width $h \equiv \Delta x$ and a time step $k \equiv \Delta t$. We then define the discrete mesh points $\left(x_{i}, t_{n}\right)$ by

$$
\begin{aligned}
x_{i} & =i h, \quad i=\cdots,-1,0,1,2, \cdots, \\
t_{n} & =n k, \quad n=0,1,2, \cdots .
\end{aligned}
$$

It is also useful to define

$$
x_{i+1 / 2}=x_{i}+\frac{h}{2}=\left(i+\frac{1}{2}\right) h .
$$

For simplicity we take a uniform mesh with $h$ and $k$ constant. Our finite-difference method we will discuss provides us with approximate values $\phi_{i}^{n} \in \mathbb{R}$ to the values of solution $\phi\left(x_{i}, t_{n}\right)$ at the discrete grid points. Here we discretize Eq. (123) as follows:

$$
\frac{\phi_{i}^{n+1}-\phi_{i}^{n}}{\Delta t}=-\frac{1}{\Delta x}\left(\tilde{f}_{i+\frac{1}{2}}-\tilde{f}_{i-\frac{1}{2}}\right)
$$

$\tilde{f}_{1 \pm \frac{1}{2}}$ denotes a numerical flux function at the cell interface $x_{i+\frac{1}{2}}$. This can be evaluated by summing up appropriate discretizations of the second and last terms of Eq. (123). Since the second term takes the form of flux, it can be discretized through the method of the Monotone Upstream Centered Schemes for Conservation Laws (MUSCL) method [7] by means of minmod limiter function [2]. Since the last term can be discretized as,

$$
\phi \frac{\partial v}{\partial x} \Rightarrow\left[\left(a_{i+\frac{1}{2}}-a_{i-\frac{1}{2}}\right) / h\right] \phi,
$$

with $a=v^{n}$, the total numerical flux can be evaluated as follows:

$$
\begin{aligned}
\tilde{f}_{i+\frac{1}{2}}= & -a_{i+\frac{1}{2}} \phi_{i} \\
& +f_{i+\frac{1}{2}}^{(u p p w)} \\
& +a_{i+\frac{1}{2}}^{+} \cdot \frac{1}{4}\left[(1+\kappa) \Phi_{i+\frac{1}{2}}^{+C}+(1-\kappa) \Phi_{i+\frac{1}{2}}^{+U}\right]
\end{aligned}
$$




$$
-a_{i+\frac{1}{2}}^{-} \cdot \frac{1}{4}\left[(1+\kappa) \Phi_{i+\frac{1}{2}}^{-C}+(1-\kappa) \Phi_{i+\frac{1}{2}}^{-U}\right] .
$$

The first term of Eq. (128) corresponds to the last term of Eq. (123), the second term $f_{i+\frac{1}{2}}^{(u p w)}$ corresponds to the first-order accurate upwind difference of the second term of Eq. (123) and the other terms are corrections of higher order accuracy. More precisely, these terms may be written as follows:

$$
f_{i+\frac{1}{2}}^{(u p w)}=a_{i+\frac{1}{2}}^{+} \phi_{i}+a_{i+\frac{1}{2}}^{-} \phi_{i+1}
$$

where

$$
a=v^{n}, \quad a^{ \pm}=v^{ \pm}=\frac{1}{2}\left(v^{n} \pm\left|v^{n}\right|\right)
$$

and $\Phi$ is defined as follows:

$$
\begin{aligned}
\Phi_{i+\frac{1}{2}}^{+C} & =\operatorname{minmod}\left[\phi_{i+1}-\phi_{i}, \beta\left(\phi_{i}-\phi_{i-1}\right)\right], \\
\Phi_{i+\frac{1}{2}}^{+U} & =\operatorname{minmod}\left[\phi_{i}-\phi_{i-1}, \beta\left(\phi_{i+1}-\phi_{i}\right)\right], \\
\Phi_{i+\frac{1}{2}}^{-C} & =\operatorname{minmod}\left[\phi_{i+1}-\phi_{i}, \beta\left(\phi_{i+2}-\phi_{i+1}\right)\right], \\
\Phi_{i+\frac{1}{2}}^{-U} & =\operatorname{minmod}\left[\phi_{i+2}-\phi_{i+1}, \beta\left(\phi_{i+1}-\phi_{i}\right)\right] .
\end{aligned}
$$

Here the minmod function is defined by

$$
\operatorname{minmod}(x, y)=\frac{1}{2}[\operatorname{sgn}(x)+\operatorname{sgn}(y)] \min (|x|,|y|) .
$$

The numerical flux $\tilde{f}_{i-\frac{1}{2}}$ is obtained by replacing subscript $i+\frac{1}{2}$ with $i-\frac{1}{2}$. In this replacement of subscript, it should be noted that the first term with the subscript replaced is not $-a_{i-\frac{1}{2}} \phi_{i-1}$ but $-a_{i-\frac{1}{2}} \phi_{i}$. When the scheme is written as

$$
\phi_{i}^{n+1}=\phi_{i}^{n}-C_{i-\frac{1}{2}}\left(\phi_{i}-\phi_{i-1}\right)+D_{i+\frac{1}{2}}\left(\phi_{i+1}-\phi_{i}\right),
$$

the conditions for the scheme to be total variation diminishing (TVD) is:

$$
C_{i+\frac{1}{2}} \geq 0, \quad D_{i+\frac{1}{2}} \geq 0, \quad C_{i+\frac{1}{2}}+D_{i+\frac{1}{2}} \leq 1 .
$$

The proof will be given later. From conditions $C_{i+\frac{1}{2}} \geq 0$ and $D_{i+\frac{1}{2}} \geq 0$, it follows that

$$
(1 \leq) \beta \leq \frac{3-\kappa}{1-\kappa} \text {. }
$$

In view of condition $C_{i+\frac{1}{2}}+D_{i+\frac{1}{2}} \leq 1$, we get

$$
\Delta t \leq \frac{\Delta x}{\left|a_{i+\frac{1}{2}}\right|+\frac{1}{4}\left(a_{i+\frac{3}{2}}^{+}-a_{i-\frac{1}{2}}^{+}\right)(\beta(1+\kappa)+1-\kappa)} .
$$


Under this condition, the scheme becomes a TVD scheme [4] for the discretization of Eq. (126). When the advective speed is constant (namely, $a=$ const), the above condition may be replaced by

$$
\Delta t \leq \frac{4}{5-\kappa+\beta(1+\kappa)} \cdot \frac{\Delta x}{|a|} .
$$

This scheme is of the third-order accuracy for the parameters $\kappa=\frac{1}{3}$ and $\beta=4$.

In the iterative implicit scheme described in the previous section, this scheme can be incorporated in the first term of Eqs. (107), (110), and (112).

5.4. TVD schemes. Proposition. When the scheme described in the previous section is written as

$$
\phi_{i}^{n+1}=\phi_{i}^{n}-C_{i-\frac{1}{2}}\left(\phi_{i}-\phi_{i-1}\right)+D_{i+\frac{1}{2}}\left(\phi_{i+1}-\phi_{i}\right),
$$

conditions for the scheme to be a total variation diminishing (TVD) are:

$$
C_{i+\frac{1}{2}} \geq 0, \quad D_{i+\frac{1}{2}} \geq 0, \quad C_{i+\frac{1}{2}}+D_{i+\frac{1}{2}} \leq 1 .
$$

PROOF. Equation (126) is rewritten as

$$
\begin{aligned}
\phi_{i}^{n+1} & =\phi_{i}^{n}-\tau\left(\tilde{f}_{i+\frac{1}{2}}-\tilde{f}_{i-\frac{1}{2}}\right) \\
& =\phi_{i}^{n}-C_{i-\frac{1}{2}} \delta \phi_{i-\frac{1}{2}}+D_{i+\frac{1}{2}} \delta \phi_{i+\frac{1}{2}},
\end{aligned}
$$

where $\delta \phi_{i-\frac{1}{2}}=\phi_{i}-\phi_{i-1}, \quad \phi_{i+\frac{1}{2}}=\phi_{i+1}-\phi_{i}$,

$$
\begin{aligned}
C_{i-\frac{1}{2}}= & \tau a_{i-\frac{1}{2}}^{+}\left[1-\frac{1+\kappa}{4} \operatorname{minmod}\left(1, \beta \frac{\delta \phi_{i-3 / 2}}{\delta \phi_{i-1 / 2}}\right)-\frac{1-\kappa}{4} \operatorname{minmod}\left(\frac{\delta \phi_{i-3 / 2}}{\delta \phi_{i-1 / 2}}, \beta\right)\right. \\
& +\frac{a_{i+1 / 2}^{+}}{a_{i-1 / 2}^{+}}\left\{\frac{1+\kappa}{4} \operatorname{minmod}\left(\frac{\delta \phi_{i+1 / 2}}{\delta \phi_{i-1 / 2}}, \beta\right)\right. \\
& \left.\left.+\frac{1-\kappa}{4} \operatorname{minmod}\left(1, \beta \frac{\delta \phi_{i+1 / 2}}{\delta \phi_{i-1 / 2}}\right)\right\}\right], \\
D_{i-\frac{1}{2}}= & -\tau a_{i-\frac{1}{2}}^{+}\left[1-\frac{1+\kappa}{4} \operatorname{minmod}\left(1, \beta \frac{\delta \phi_{i+3 / 2}}{\delta \phi_{i+1 / 2}}\right)-\frac{1-\kappa}{4} \operatorname{minmod}\left(\frac{\delta \phi_{i+3 / 2}}{\delta \phi_{i+1 / 2}}, \beta\right)\right. \\
& +\frac{a_{i-1 / 2}^{-}}{a_{i+1 / 2}^{-}}\left\{\frac{1+\kappa}{4} \operatorname{minmod}\left(\frac{\delta \phi_{i-1 / 2}}{\delta \phi_{i+1 / 2}}, \beta\right)\right. \\
& \left.\left.+\frac{1-\kappa}{4} \operatorname{minmod}\left(1, \beta \frac{\delta \phi_{i-1 / 2}}{\delta \phi_{i+1 / 2}}\right)\right\}\right] .
\end{aligned}
$$

Using the conditions below,

$$
C_{i+1 / 2} \geq 0, \quad D_{i+1 / 2} \geq 0, \quad C_{i+1 / 2}+D_{i+1 / 2} \leq 1,
$$


we get the following estimate:

$$
\begin{aligned}
T V\left(\phi^{n+1}\right)= & \sum_{i}^{M}\left|\phi_{i+1}^{n+1}-\phi_{i}^{n+1}\right| \\
= & \sum_{i}^{M} \mid \phi_{i+1}^{n}-\phi_{i}^{n}+D_{i+3 / 2} \delta \phi_{i+3 / 2}-C_{i+1 / 2} \delta \phi_{i+1 / 2} \\
& -D_{i+1 / 2} \delta \phi_{i+1 / 2}+C_{i-1 / 2} \delta \phi_{i-1 / 2} \mid \\
\leq & \sum_{i}^{M}\left[\left\{1-\left(C_{i+1 / 2}+D_{i+1 / 2}\right)\right\}\left|\delta \phi_{i+1 / 2}\right|\right. \\
& \left.+C_{i-1 / 2}\left|\delta \phi_{i-1 / 2}\right|+D_{i+3 / 2}\left|\delta \phi_{i+3 / 2}\right|\right] .
\end{aligned}
$$

Letting $M \rightarrow \infty$, for $\operatorname{supp}(\phi) \subset[-M, M]$, we obtain

$$
\begin{aligned}
\sum_{i}^{M}\left(C_{i-1 / 2}\left|\delta \phi_{i-1 / 2}\right|\right) & =\sum_{i}^{M}\left(C_{i+1 / 2}\left|\delta \phi_{i+1 / 2}\right|\right) \\
\sum_{i}^{M}\left(D_{i+3 / 2}\left|\delta \phi_{i+3 / 2}\right|\right) & =\sum_{i}^{M}\left(D_{i+1 / 2}\left|\delta \phi_{i+1 / 2}\right| .\right.
\end{aligned}
$$

Therefore,

$$
\begin{aligned}
T V\left(\phi^{n+1}\right) \leq & \sum_{i}^{M}\left[\left\{1-\left(C_{i+1 / 2}+D_{i+1 / 2}\right)\right\}\left|\delta \phi_{i+1 / 2}\right|\right. \\
& +C_{i+1 / 2}\left|\delta \phi_{i+1 / 2}\right|+D_{i+1 / 2}\left|\delta \phi_{i+1 / 2}\right| \\
= & \sum_{i}^{M}\left|\delta \phi_{i+1 / 2}\right|=T V\left(\phi^{n}\right) .
\end{aligned}
$$

\section{Numerical results}

In this section, the proposed numerical scheme is discussed by computing the following three types of fluid flow problems.

1. Lid driven flows in the square cavity

2. Natural convection flows in the square cavity

3. Premixed laminar combustion of hydrogen and bromine

Problem 1 is what calculates the flow field in the square cavity driven by the shear force generated when the top wall moves towards the right with uniform velocity. Here it is assumed that initial gas is filled in the cavity and that the top wall of the cavity is moving at a constant 
speed. This problem has served over and over again as a model problem for testing and evaluating numerical techniques for incompressible fluid flows, in spite of the singularities at the two corners. Here, this problem is taken up as a validation problem for the numerical scheme for fluid flow motion without the density change. Problem 2 is the flow fields advected by the buoyancy which is caused by the density change due to the change in temperature under the gravity. As initial conditions we assume that the square cavity is filled with gas, and that the temperature difference of $10{ }^{\circ} \mathrm{C}$ is given to the right and left walls. Here, this problem is taken up as a validation problem for the numerical scheme to describe natural advection of fluid in case of small ratios of the density change to the temperature change. The Boussinesq approximation has been employed to such kind of problems so far. Our numerical scheme involves methods for incompressible fluid flows, fluid flows to which the Boussinesq approximation is applicable, and fluid flows with large temperature difference that we have experienced in the combustion problems. The Problem 3 is concerned with the numerical methods for computing the flow fields with chemical reaction. We numerically solve the propagation of the flame surface formed by igniting a part of premixed gases moving with uniform velocity. In this case, the temperature change becomes $1000^{\circ} \mathrm{C}$ or more according to the combustion.

6.1. Lid-driven square cavity flows without density variation. Schematic of the problem is shown in Fig. 1. For all walls, an adiabatic condition is imposed on the temperature, non-slip velocity condition is applied to the bottom, right and left walls. The top wall moves with uniform velocity. The size of cavity and velocity value are set so that Reynolds number is equal to the given conditions. As initial conditions, the pressure is set to be $0.1 \mathrm{MPa}$, and the temperature is set to be $300 \mathrm{~K}$. The Reynolds numbers based on the top-wall velocity are $R e=1,000,3,200$, and 7,500. The CFL number $v \Delta t / \Delta x$ is set to be 0.5 and the mesh size is $101 \times 101$ for all calculations. In Fig. 2, the computed velocity profiles for vertical and horizontal lines passing through the geometric center of the cavity. In the figure, the computed $u$-velocity along vertical line and $v$-velocity along horizontal line through geometric center for $R e=1,000$ are shown with the results taken from Ghia et al. [3]. Although their results were calculated using a vorticity-stream function method that is only applicable to the two-dimensional incompressible flow fields, their results have been well referred by many researchers as an example of high-accurate solutions and have been used for validation of numerical schemes by comparing with their numerical results. Our results agree well with the results of Ghia et al. as shown in the velocity profiles presented. The computed contours of stream function for $R e=1,000,3,200$, and 7,500 are shown with results of Ghia et al. in Figs. 3, 4, and 5, respectively. Although the figures are not depicted using the same values of contour lines, we find that both results agree well in a qualitative way. Figure 3 for $R e=1,000$ shows that the calculation capture the corner vortices formated at two respective bottom corners of the cavity. It is seen that for $R e=3,200$ the third vortex appears around the top-left corner of the cavity in Fig. 4. In Fig. 5 it is observed that the fourth vortex forms at the bottom-right corner of the cavity. It can be mentioned from these computational results 


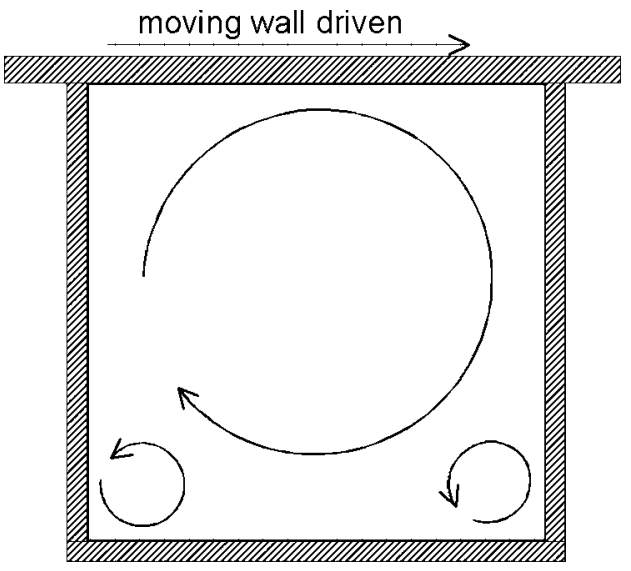

FIGURE 1. Schematic of wall shear driven flows in a square cavity.

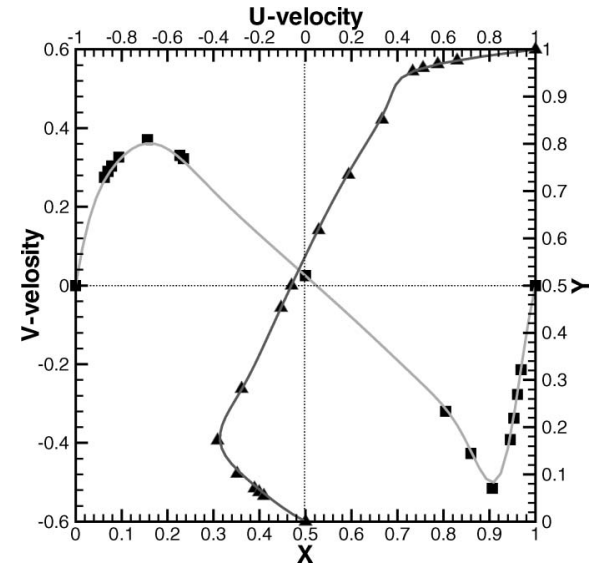

FIGURE 2. Comparison with the results of Ghia, U. et al.

presented here that our results capture the flow feature for each Reynolds number as well as the Ghia's results in a satisfactory way.

6.2. Natural convection in square cavity flows due to buoyancy. Computations of natural advection in the square cavity flows driven by buoyancy arising from the density change due to the change in temperature under gravity are made. This numerical problem was first suggested by Jones [6] as test case for suitable validation for numerical techniques. Although the Boussinesq approximation is applicable to this problem, we here employ the low-Mach number approximation in a way similar to problem 1. The schematic diagram of 


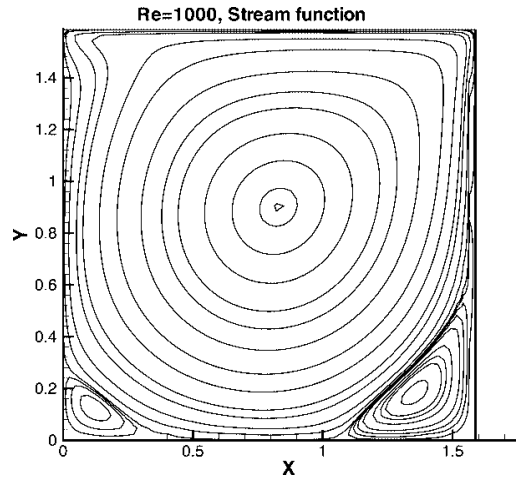

(a) Present result

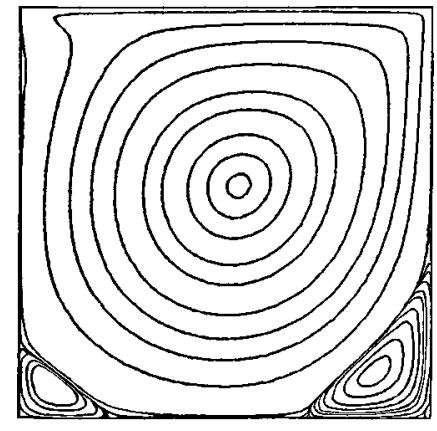

(b) Result taken from [3]

Figure 3. Comparison of stream function contours in lid-driven square cavity flows at $R e=1,000$.

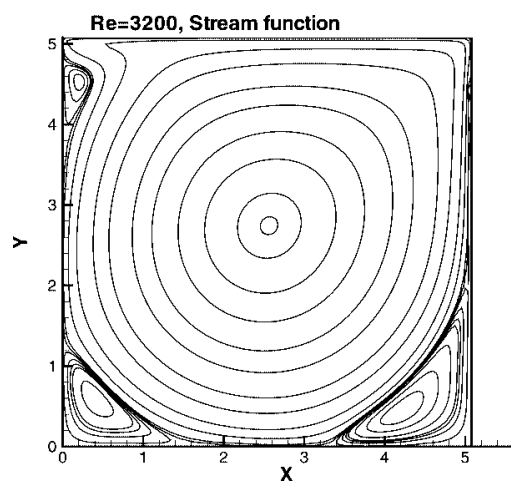

(a) Present result

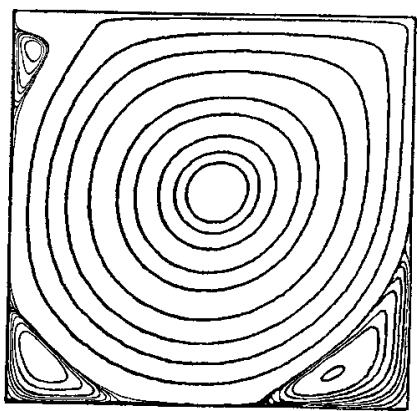

(b) Results taken from [3]

FIGURE 4. Comparison of stream function contours in lid-driven square cavity flows at $R e=3,200$.

the problem is shown in Fig. 6. As the wall boundary conditions for the temperature, temperature of $305 \mathrm{~K}$ is set on the vertical-left wall, and that of $295 \mathrm{~K}$ is set on the vertical-right wall. The adiabatic wall conditions, that corresponds to the homogeneous Neumann condition for the temperature, are assumed on the bottom and top walls. We suppose that the gas is air and the Rayleigh number $R a$ is 10,000 , so that the size of cavity $L$ can be determined using the following equation:

$$
R a=\frac{\beta|\mathbf{g}| \Delta T L^{3}}{\nu k /\left(C_{p} \rho\right)}
$$

where $\beta$ denotes the thermal coefficient of volumetric expansion, $v$ means the kinematic viscosity, $k$ is the thermal conductivity, and $C_{p}$ stand for the specific heat at constant pressure. As initial states, $0.1 \mathrm{MPa}$ of the pressure and $300 \mathrm{~K}$ of the temperature are assumed. The 


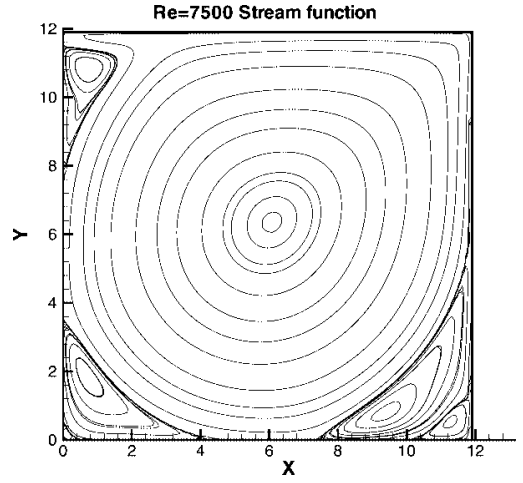

(a) Present result

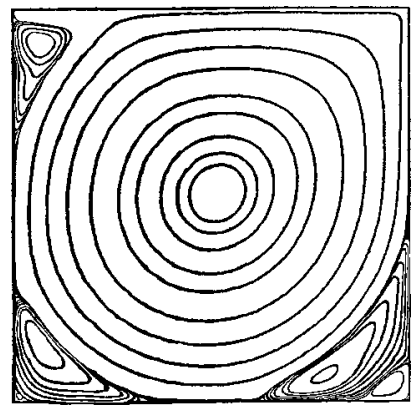

(b) Results taken from [3]

FIGURE 5. Comparison of stream function contours in lid-driven square cavity flows at $R e=7,500$.

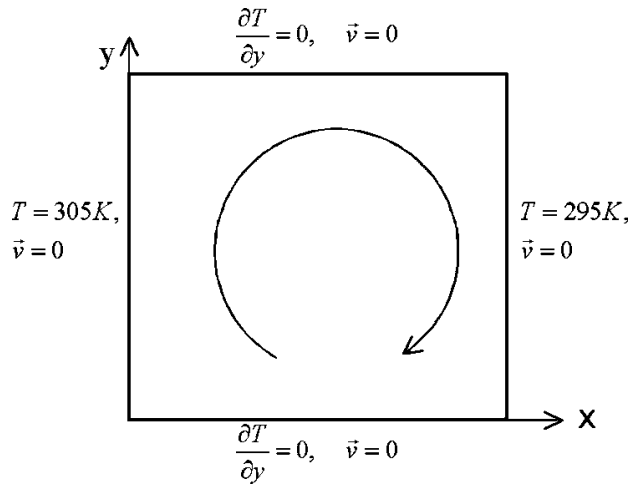

FIGURE 6. Schematic of natural convection flows in a square cavity.

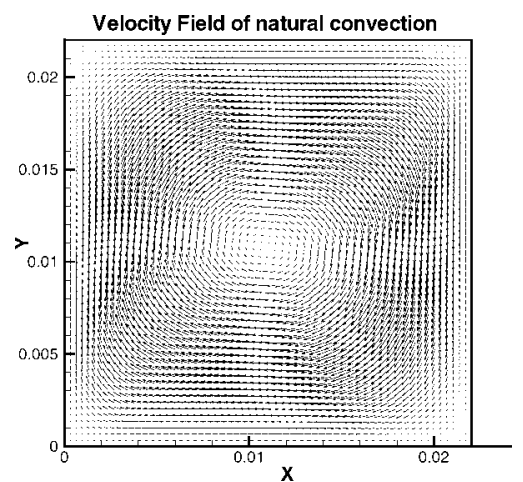

FIGURE 7. Velocity field in natural convection at $R a=10,000$.

flow fields at steady state solution are shown in Figs. 7, 8 and 9 for the velocity, temperature, and density, respectively. These results agree the results with classical Boussinesq equations published in [12]. Accordingly, the present scheme is confirmed to be applicable to the flows with density variation due to variation in temperature.

6.3. Chemically reacting flows. In order to verify the codes, computation of the premixed combustion of hydrogen and bromine was performed. This problem has been investigated by Spalding and Stephenson [11] in which the following four-stage elementary reactions are taken into account:

(1) $B r_{2}+M \leftrightarrow 2 B r+M$

(2) $H_{2}+M \leftrightarrow 2 H+M$ 


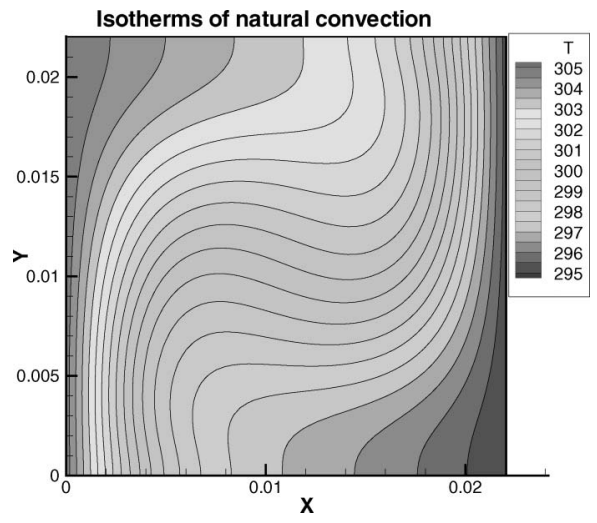

FIGURE 8. Isotherms in natural convection at $R a=10,000$.

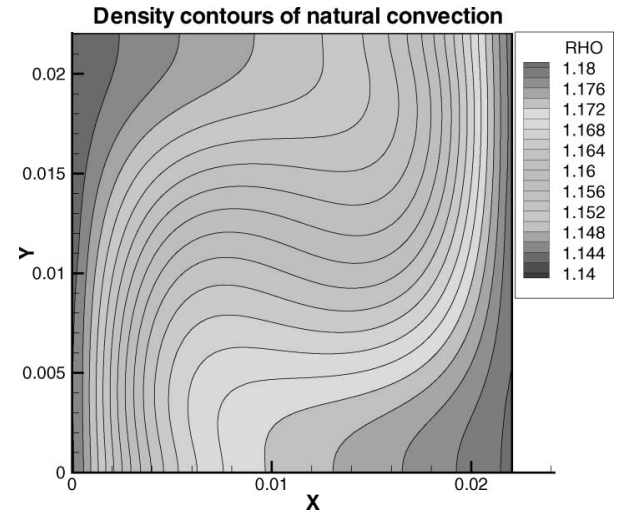

FIGURE 9. Density contours in natural convection at $R a=10,000$.

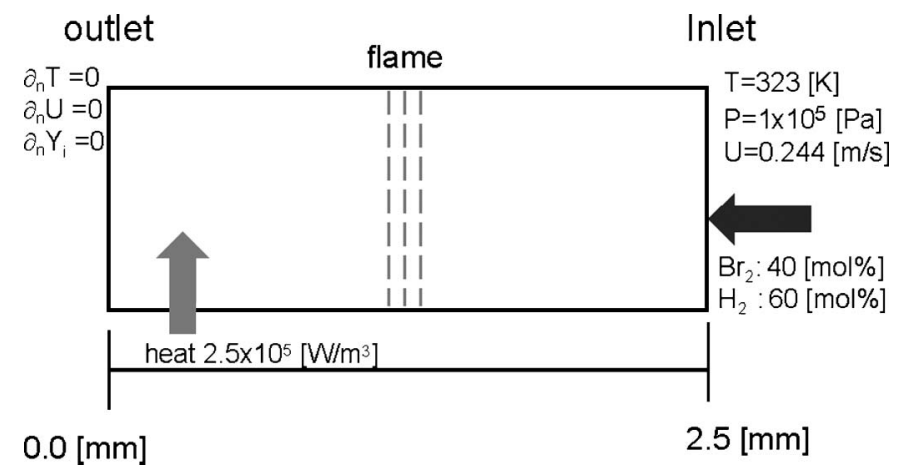

FIGURE 10. Schematic model of laminar flame propagation of combustion of hydrogen and bromine.

$$
\begin{array}{ll}
\text { (3) } & \mathrm{Br}+\mathrm{H}_{2} \leftrightarrow \mathrm{HBr}+\mathrm{H} \\
\text { (4) } \mathrm{H}+\mathrm{Br}_{2} \leftrightarrow \mathrm{HBr}+\mathrm{Br}
\end{array}
$$

The schematic of the computational model is shown in Fig. 10.

6.3.1. Calculation condition. The reaction rate constant for the $i$-th stage, $k_{i}$, is computed by means of Arrhenius' law, as follows:

$$
k_{i}=B_{i} T^{\alpha_{i}} \exp \left(\frac{-E_{i}}{R T}\right) \quad\left[\left(\mathrm{mol} / \mathrm{m}^{3}\right)^{1-\mathrm{n}^{-1}}\right]
$$

where $B_{i}$ is the frequency factor $\left[\left(\mathrm{mol} / \mathrm{m}^{3}\right)^{1-\mathrm{n}} \mathrm{K}^{-\alpha_{\mathrm{i}}} \mathrm{s}^{-1}\right], E_{i}$ means the activation energy [J/mol] $\alpha_{i}$ denotes the temperature dependent parameter, $T$ stands for the absolute temperature $[\mathrm{K}], R$ represents the universal gas constant $[\mathrm{J} / \mathrm{mol} \mathrm{K}]=8.314[\mathrm{~J} /(\mathrm{mol} \mathrm{K})]$, and $n$ denotes 
the reaction index $n=a+b$. The Arrhenius parameters used in this study are shown in Table 1 below. The material properties for chemical species are calculated through the following temperature dependent formula.

Diffusion coefficient $D$ :

$$
D=D_{0}\left(\frac{T}{T_{r e f}}\right)^{1.67}
$$

Thermal conductivity $\lambda$ :

$$
\lambda=\lambda_{0}\left(\frac{T}{T_{r e f}}\right)^{0.67}
$$

where $T_{r e f}=323[\mathrm{~K}]$, the parameter $D_{0}$ in the equation of the diffusion coefficient and parameter $\lambda_{0}$ in the equation of the thermal conductivity are listed in Table 2 below. The same value of $C_{p}$ is used for all species as in the paper by Spalding and Stephenson [11].

$$
C_{p}=530.86[\mathrm{~J} /(\mathrm{kg} \mathrm{K})] .
$$

6.3.2. Initial and boundary conditions. As initial conditions, the velocity is set to be zero $(u=0[\mathrm{~m} / \mathrm{s}])$, the temperature is set to be $49.85^{\circ} \mathrm{C}$, the pressure is set to be $1 \times 10^{5}[\mathrm{~Pa}]$, the mole fraction of bromine is set to be $0.4\left(X_{B r_{2}}=0.4\right)$, and the mole fraction of hydrogen is set to be $0.6\left(X_{H_{2}}=0.6\right)$ in the whole computational domain. More precisely, the following boundary conditions are used:

TABLE 1. Chemical reaction parameters.

\begin{tabular}{|l|c|r|r|c|c|r|}
\hline Reaction & \multicolumn{3}{|c|}{ Forward Reaction } & \multicolumn{3}{c|}{ Backward Reaction } \\
\hline Arrhenius parameters & $B_{i}$ & \multicolumn{1}{|c|}{$\alpha_{i}$} & \multicolumn{1}{c|}{$E_{i}$} & $B_{i}$ & $\alpha_{i}$ & $E_{i}$ \\
\hline \hline (1) $\mathrm{Br}_{2}+M \leftrightarrow 2 B r+M$ & $7.03 \times 10^{11}$ & -0.5 & 196700 & $3.63 \times 10^{3}$ & 0.0 & 0 \\
\hline (2) $H_{2}+M \leftrightarrow 2 H+M$ & $7.63 \times 10^{13}$ & -1.0 & 452100 & $3.63 \times 10^{3}$ & 0.0 & 0 \\
\hline (3) $B r+H_{2} \leftrightarrow H B r+H$ & $3.46 \times 10^{4}$ & 1.0 & 69490 & $9.06 \times 10^{5}$ & 0.5 & 7158 \\
\hline (4) $H+B r_{2} \leftrightarrow H B r+B r$ & $6.42 \times 10^{6}$ & 0.5 & 4646 & $6.52 \times 10^{4}$ & 1.0 & 178300 \\
\hline
\end{tabular}

TABLE 2. Properties of species.

\begin{tabular}{|l|r|r|r|r|}
\hline Species & Molecular Mass $[\mathrm{kg} / \mathrm{mol}]$ & Enthalpy $[\mathrm{J} / \mathrm{mol}]$ & $D_{0}\left[\mathrm{~m}^{2} / \mathrm{s}\right]$ & $\lambda_{0}[\mathrm{~W} / \mathrm{mK}]$ \\
\hline $\mathrm{H}_{2}$ & $2.016 \times 10^{-3}$ & 0.0 & $1.01 \times 10^{-5}$ & $3.34 \times 10^{-2}$ \\
\hline $\mathrm{Br} r_{2}$ & $159.8 \times 10^{-3}$ & $3.09 \times 10^{4}$ & $1.01 \times 10^{-5}$ & $3.34 \times 10^{-2}$ \\
\hline $\mathrm{HBr}$ & $80.908 \times 10^{-3}$ & $-3.66 \times 10^{4}$ & $1.01 \times 10^{-5}$ & $3.34 \times 10^{-2}$ \\
\hline $\mathrm{H}$ & $1.008 \times 10^{-3}$ & $2.18 \times 10^{5}$ & $1.01 \times 10^{-5}$ & $3.34 \times 10^{-2}$ \\
\hline $\mathrm{Br}$ & $79.90 \times 10^{-3}$ & $1.12 \times 10^{5}$ & $1.01 \times 10^{-5}$ & $3.34 \times 10^{-2}$ \\
\hline
\end{tabular}


On the inlet boundary:

$$
\begin{aligned}
& u=0.244[\mathrm{~m} / \mathrm{s}] \\
& T=49.85\left[{ }^{\circ} \mathrm{C}\right] \\
& p_{n}=0 \\
& X_{B r_{2}}=0.4 \\
& X_{H_{2}}=0.6 \\
& X_{H B r n}=0 \\
& X_{H n}=0 \\
& X_{B r n}=0
\end{aligned}
$$

On the outlet boundary:

$$
\begin{aligned}
& u_{n}=0 \\
& T_{n}=0 \\
& X_{B r_{2 n}}=0 \\
& X_{H_{2 n}}=0 \\
& p=1 \times 10^{5}[\mathrm{~Pa}] \\
& X_{H n}=0 \\
& X_{B r n}=0
\end{aligned}
$$

Here subscript " $n$ " denotes the derivative in the normal direction to the boundary.

6.3.3. Computational results. At the beginning of computation, heat source is given nearby outlet as follows.

$$
S(x, 0)=2.5 \times 10^{10}\left[\mathrm{~W} / \mathrm{m}^{3}\right] \quad x \in\left[0.75 \times 10^{-4}, 1 \times 10^{-4}\right][\mathrm{m}] .
$$

As time goes by, the flame surface has propagated toward the center of the computational domain. When the flame reached to the center, the heat source has been removed. After then, the flame propagation has stopped and kept the position. The computation has been made until the temperature and species concentrations reached the steady state.

The computed species concentrations and temperature are shown in Fig. 11. From the right boundary, unburnt gases come into the computational domain, and the flame front is formed around the center of domain. In order that gases come from the inlet boundary with the velocity of $0.244[\mathrm{~m} / \mathrm{s}]$, the flame front moves with the relative velocity to the coming gases. Since the mainstream velocity of $0.244[\mathrm{~m} / \mathrm{s}]$ is equal to the laminar flame propagation speed on this combustion, the flame propagation stops around center of the domain after the removement of the heat source. It is seen that the radical species, $\mathrm{H}$ and $\mathrm{Br}$ have the peak values just behind flame front surface. The results taken from Spalding and Stephenson [11] is also shown in Fig. 12. Our result is in good agreement with that of Spalding and Stephenson. Thus, it is confirmed that the results obtained through our numerical scheme are reasonable.

\section{Concluding remarks}

The mathematical model based on the low-Mach-number approximation is presented and a new numerical method is proposed. Our numerical scheme consists of an iterative implicit time evolution of the second order accuracy and a spatial discretization with the TVD property of high accuracy. The implicit iteration algorithm for time evolution is effective for not only numerical stability but also for the use of TVD discretization of the nonlinear convective terms in the implicit scheme.

In order to validate the numerical scheme, the lid-driven cavity flow, natural convection in the square cavity, and the flame propagation problem are solved as appropriate validation 


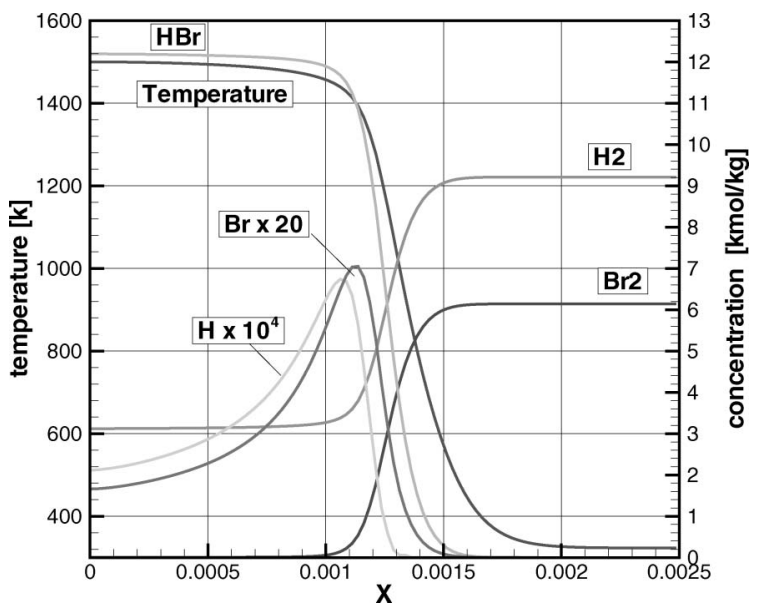

FIGURE 11. Profiles of computational results of concentration and temperature of the species.

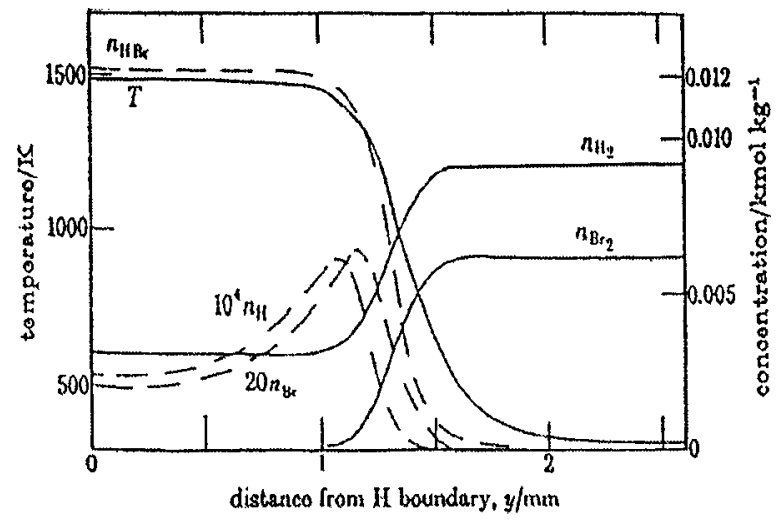

FIGURE 12. Results cited from Spalding and Stephenson [11].

of test cases for the incompressible flows, density variation flows with small temperature fluctuation and also chemically reacting flows with difference in large temperature, respectively. From the results for the validation of test cases, our numerical method is validated for a wide range of flows with density variation.

\section{References}

[ 1 ] R. B. Bird, W. E. Stewart and E. N. Lightfoot, Transport Phenomena, John Wiley \& Sons, Inc. (1960).

[ 2 ] S. R. ChAKRAVARTHY and S. OShER, A new class of high accuracy TVD scheme for hyperbolic conservation laws, AIAA Paper 85-0363 (1985). 
[ 3 ] U. Ghia, K. N. GhiA and C. T. Shin, High-Re solutions for incompressible flow using the Navier-Stokes equations and a multi-grid method, Journal Computational Physics 48 (1982), 387-411.

[ 4 ] A. HARTEN, On a class of high resolution Total-Variation-Stable finite-difference schemes, SIAM Journal of Numerical Analysis 21 (1) (1984), 1-12.

[ 5 ] R.KLEIN, Semi-implicit extension of Gudonov-type scheme based on low Mach number asymptotic I: Onedimensional flow, Journal Computational Physics 121 (1995), 213-237.

[ 6 ] I. P. JONES, A comparison problem for numerical methods in fluid dynamics, The 'Double-Glazing' problem, in Numerical Methods in Thermal Problems, Proceedings of the First International Conference (1979) 338348.

[ 7 ] B. VAN LEER, Toward the ultimate conservative difference scheme. 4, A new approach to numerical convection, Journal of Computational Physics 23 (1977), 276-299.

[8] A. MAJDA, Compressible fluid flow and systems of conservation laws in several space variable, Springer, New York (1984).

[9] H. N. Najm, P. S. Wyckoff and O. M. Knio, A Semi-implicit Numerical Scheme for Reacting Flow, Journal of Computational Physics 143 (1998), 381-402.

[10] C. M. RHIE and W. L. CHOw, A numerical study of the turbulent flow past an isolated airfoil with trailing edge separation, AIAA Journal 21 (11) (1983), 1525-1532.

[11] D. B. SpAlding and P. L. Stephenson, Laminar flame propagation in hydrogen+bromine mixtures, Proceedings of Royal Society of London A324, 315 (1971).

[12] G. DE VAhl Daivis and I. P. Jones, Natural Convection in a Square Cavity. A Comparison Exercise, International Journal for Numerical Method in Fluids 3, 1983, 227-248.

[13] C. R. WILKE, Journal of Chemical Physics 18, 517 (1950).

Present Address:

Fundamental Technology Research Center, Honda R\&D Co., Ltd.

WAKO-SHI, SAITAMA, 351-0193 JAPAN.

e-mail: Toshiyuki_Arima@n.f.rd.honda.co.jp 